\title{
Temperature and Weight Monitoring of the Apis Cerana Bee Colony Indonesia
}

\author{
Armands Kviesis ${ }^{1}$, ${ }^{*}$ Aleksejs Zacepins ${ }^{1}$, Vitalijs Komasilovs ${ }^{1}$, Amanda Manggiasih Paramita ${ }^{2}$, Fakhri \\ Rido Muhammad ${ }^{2}$ \\ ${ }^{1}$ Department of Computer Systems, Faculty of Information Technologies, Latvia University of Life Sciences \\ and Technologies, Jelgava, Latvia \\ ${ }^{2}$ Labtek Indie, Bandung, West Java, Indonesia
}

\begin{abstract}
Remote and automatic monitoring of two Apis Cerana bee colonies was conducted in Indonesia to demonstrate precision beekeeping approach in that region. Successful implementation of the precision beekeeping system includes development of the bee colony monitoring hardware and software for data collection, analysis and visualisation. This paper focuses on development and installation of such systems at the private apiary in Indonesia. For bee colony monitoring at the apiary a developed monitoring unit was used, which is based on ESP microchip, and for the data storage SAMS data warehouse was used. The monitoring results showed that the choice of the location of the temperature sensor is important, as the temperature at the hive sides changes synchronously with the outside temperature. Also, feedback from the beekeeper is collected to further improve the system and monitoring process. This research is conducted within the SAMS - Smart Apiculture Management Services project, which is funded by the European Union within the H2020-ICT-39-2016-2017 call and with close collaboration with the local private beekeeper. To find out more, visit the project website https://sams-project.eu/.
\end{abstract}

Keywords: Precision beekeeping, SAMS, bee colony monitoring, temperature and weight monitoring.

\section{Introduction}

Asian honey bees, Apis Cerana (A. cerana), are honey bees from southern and south-eastern Asia with a variety of sub-species found in different Asian countries including Indonesia (Hyatt, 2012). Asian honey bee Apis Cerana is known for their gentle temperament and easy handling. To assess the colony strength, existence of pests, parasites and diseases beekeepers must open the hive and visually inspect it on a regular basis. (Brodschneider et al., 2018, 2016; Delaplane, Van Der Steen, \& Guzman-Novoa, 2013; Gray et al., 2019; Van Der Zee et al., 2012). However, manual monitoring of beehives is a timeconsuming process for beekeepers and stressful to the bee colonies. Time-consumption even increases with the distance of the beekeeping sites to the homesteads, as bee colonies are frequently placed in rural areas, far from cities, in jungles or mountain areas, so every inspection also incurs travel costs to beekeepers.
To facilitate and improve the hive management procedure, the implementation of smart apiary management services is believed to be the future of modern beekeeping (Bencsik et al., 2011; EdwardsMurphy et al., 2015; Meikle \& Holst, 2015; Zacepins et al., 2016). For this approach, information communication and technology (ICT) based on remote sensing tools to monitor the bee colony's health and productivity are used (Zacepins et al., 2015). While precision beekeeping approach is already widely used in Europe, the USA and other developed countries, it is concluded that in Indonesia, automated and remote bee colony monitoring is only on its starting point, and temperature, weight monitoring are not widely practiced. There are only few articles on the digital monitoring of Apis Cerana parameters. For example, some authors investigated the variances of the temperature, relative humidity and $\mathrm{CO} 2$ concentration among brood combs in the Apis cerana colony, by

\footnotetext{
* Corresponding Author's email: aleksejs.zacepins@1lu.lv
} 
using the electric digital thermometer (Guanhuang et al., 1999). Others conducted a systematic observation and research on the overwintering and size dynamic characters of Apis cerana in south and west mountainous area of Anhui Province in China in 1998-2003 (Yu \& Han, 2003).

Based on temperature dynamics, different bee colony states can be identified (like swarming, brood rearing, broodless state). And based on bee colony weight dynamics, food consumption and amount of food storage can be evaluated and lack of resources can be predicted. For example, abnormal food consumption can indicate some unwanted behaviour of the bee colony. High food consumption during the passive period could indicate increased activity of the colony, possible brood rearing. Too early brood rearing is not always welcomed, as to the moment when the colony is strong, there could be insufficient foraging resources. In addition, bee colonies tend to abscond in Indonesia. Biological definition of the absconding comprises the departure of all adult bees of a colony from their nest, leaving behind whatever brood and stores are in it. Absconding may be due to shortage of food, disturbance, or to other adverse circumstances (Crane, 1990). Therefore, a remote monitoring system together with data analysis solutions can alert the beekeeper when the bees abscond.

This research is done within the SAMS project in collaboration with private Indonesian beekeeper. A combined biological, sociological and technical approach is made within the SAMS (Smart Apiculture Management Services) project. It enhances international cooperation of ICT and sustainable agriculture between EU and developing countries in pursuit of the EU commitment to the UN Sustainable Development Goal "End hunger, achieve food security and improved nutrition and promote sustainable agriculture". Main objectives of SAMS are to develop, refine and implement an open source remote sensing technology for monitoring the health and

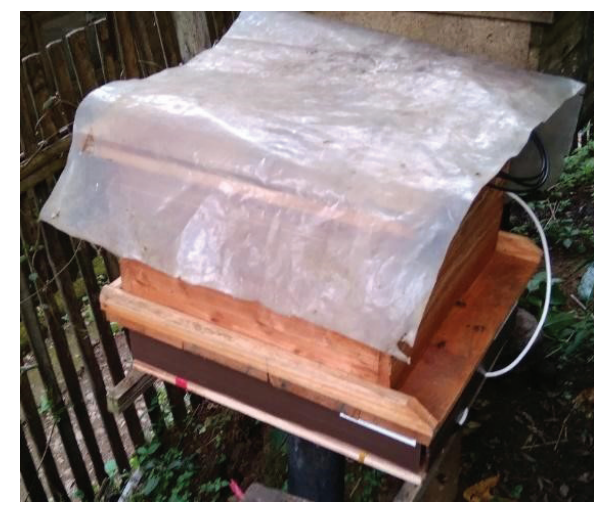

Figure 1. Modern beehive used in the experiment. productivity of bee colonies and to foster the regional added benefit and gender equality in employment. The SAMS project focuses on beekeeping in Ethiopia (Demisew, 2016; Negash \& Greiling, 2017; Wakjira \& Alemayehu, 2019) and Indonesia (Gratzer et al., 2019) as in those countries there is a huge beekeeping potential that is not fully discovered yet.

The aim of this study is to assess the feasibility of using precision beekeeping for bee colony temperature and weight monitoring of Apis Cerana in Indonesia, as well as to demonstrate that remote and automated monitoring of Apis Cerana is possible, and it can provide valuable information for the local beekeepers. This article presents the first results of measuring Apic Cerana temperature and weight during summer period.

\section{Materials and Methods}

\section{Bee colonies}

Since this study focuses on the evaluation and feasibility of the proposed monitoring system, only two bee colonies of Apis Cerana were subjected to experiments in the field. One bee colony is placed in a modern beehive (Figure 1).

The modern hive is made of wood with the outer size of $40 \mathrm{~cm}$ length, $30 \mathrm{~cm}$ width and $25 \mathrm{~cm}$ height with wall thickness of $1,5 \mathrm{~cm}$. The second bee colony is placed in a frameless beehive which is also called "Golodog" with the same hive dimensions (Figure 2). Golodog type of modern beehives is actually a simple modern beehive that is used as a bee colony trap box. Beekeepers use this box to acquire new bee colonies and move the colony in the standard (with frames) modern beehive once the bee colony has settled, but sometimes they let the bee colony stay in this hive anyway.

Bee species under monitoring is Apis Cerana Cerana.

Location of the bee colonies

Experiment was carried out in an open environment at the private Madu Maribaya Apiary. This apiary

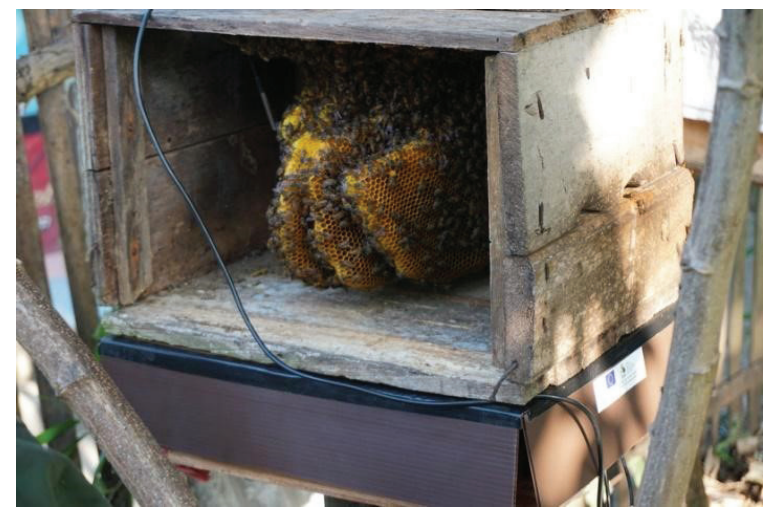

Figure 2. Frameless hive "Golodog". 
is owned by Pak Koswara in Maribaya, West Java, Indonesia.

Status of the colonies

Based on the visual inspection of the hives, the beekeeper concludes that both colonies are in good condition. This is evaluated by the experience of the beekeeper by his own evaluation procedure. Procedure includes several basic actions: observation if the bees are still there, if the brood is present and the amount of it is sufficient, if the queen is present, and if the colony produce honey. In the case if all indicators are present, a conclusion about the bees condition can be made.

SAMS bee colony monitoring device

Two (2) bee colonies were equipped with a SAMS hive monitoring system based on the NodeMCU electronic platform (ESP8266 microchip) (Zacepins et al., 2020), Figure 3.

For weight monitoring, a single-point load cell Bosche H30A was used. For the bee colony temperature monitoring DS18S20 1-Wire ${ }^{\circledR}$ sensors were used.

Architecture of the developed hardware platform is shown in Figure 4:

Temperature sensors were placed in three positions inside the hive: in the middle frame, in the most right frame and in the most left frame (Figure 5). Authors chose different sensor positions with the aim to compare the temperature dynamics and then conclude about the appropriate sensor position for the reliable temperature measurements.

Environmental conditions (temperature and humidity) were monitored using a DHT22 sensor. The NodeMCU platform were powered by a power bank. For the research purpose, data was collected each two (2) minutes and sent to the remote SAMS data warehouse. For internet connection, a mobile Huawei router was used, which was connected to the electric grid at the beekeeper's house. Distance from the router to the monitoring nodes is around $10 \mathrm{~m}$. As mobile connection at the apiary location was poor, interruption of Internet connection occurred and some data points were lost during the monitoring period, but this does not affect the overall conclusion making and monitoring process itself.

The first bee colony monitoring took place on 04.07.2020 and is still ongoing.

The second bee colony monitoring took place on 01.08.2020 and is still ongoing.

SAMS data warehouse

The SAMS Data Warehouse (DW) is a universal system, which can operate with different data inputs,

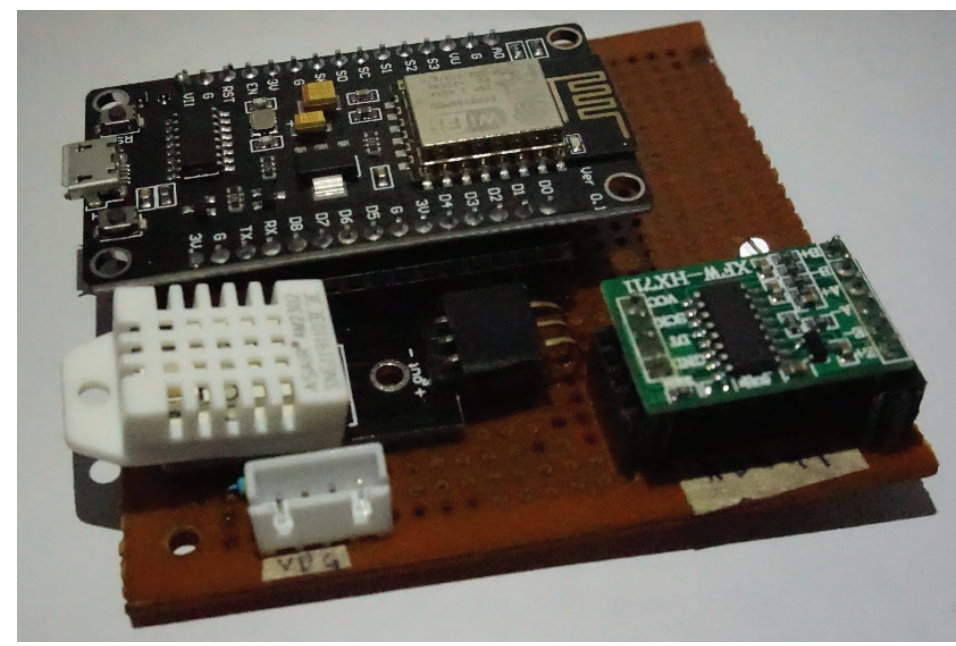

Figure 3. NodeMCU based hardware system.

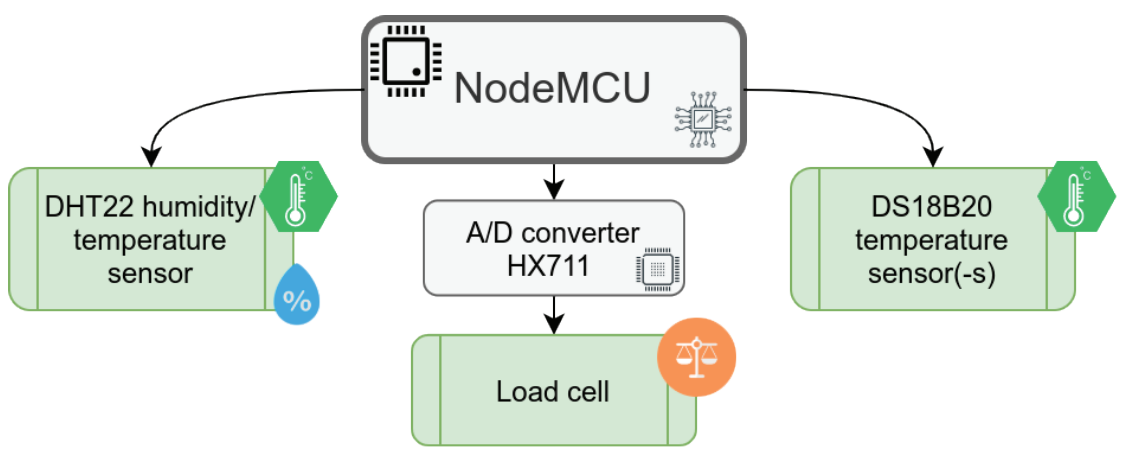

Figure 4. Hardware platform architecture. 


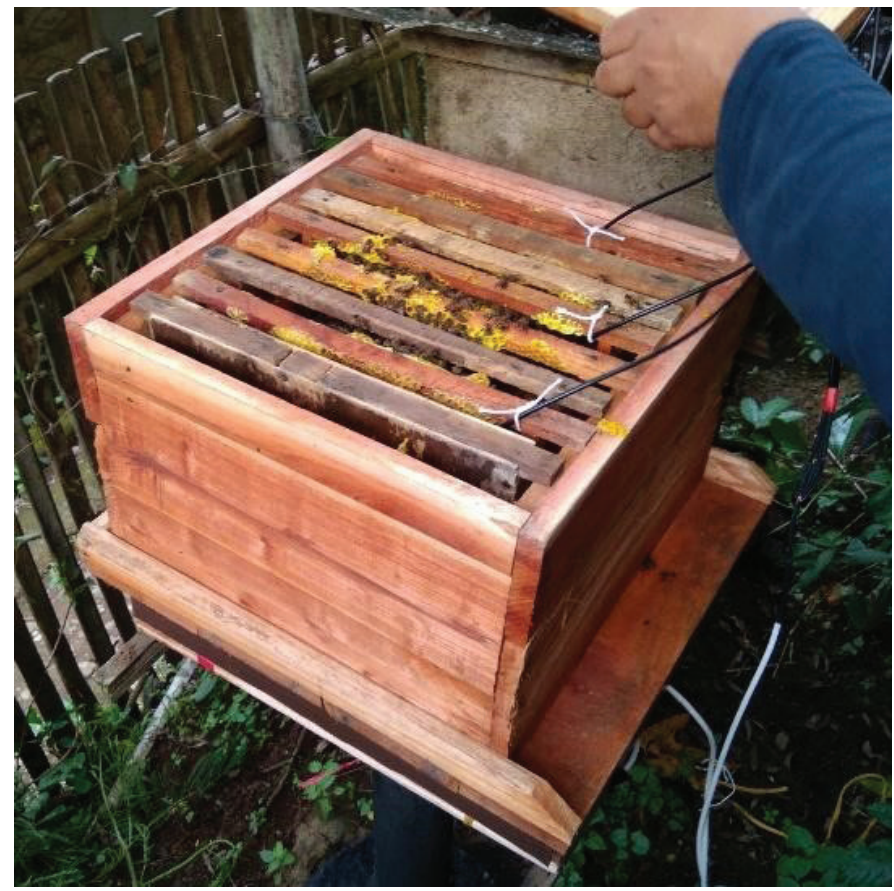

Figure 5. Sensor placement in the modern beehive.

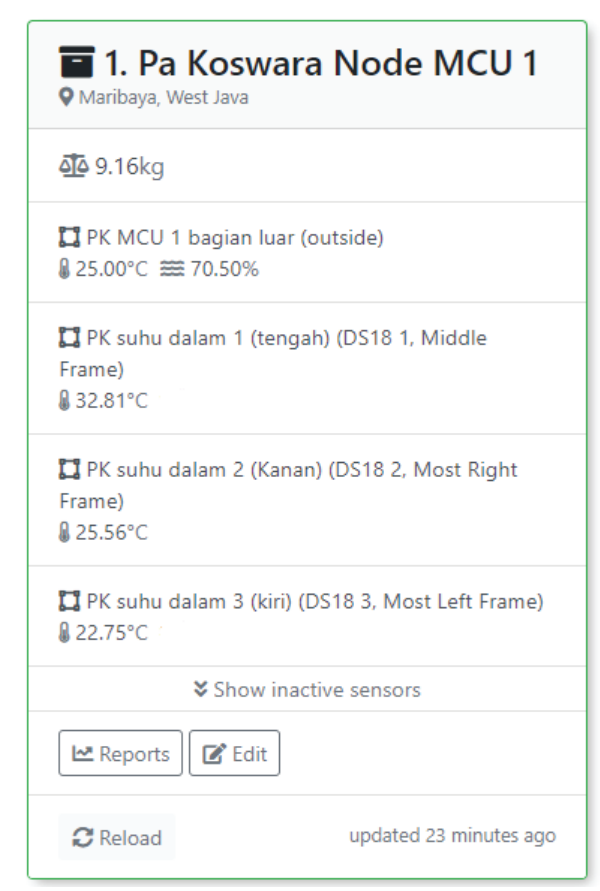

Figure 6. Summary of the bee colony data. such as direct data transfer from bee colony monitoring devices as well as manual data upload, which has flexible data processing algorithms based on the incoming data sets. SAMS DW can be connected not only with SAMS monitoring devices, but also with other general available bee colony monitoring systems. The SAMS Data Warehouse is already developed and is a fully operational platform (https:// sams.science.itf.llu.lv/). It can easily be accessed from any web browser. All measured data is stored in the SAMS DW for further analysis and report creation.

The screenshot below demonstrates how the summary of the colony monitoring is shown to the beekeeper in the SAMS DW in real-time (Figure 6):

\section{Results and Discussion}

\section{Temperature monitoring}

The performed monitoring results showed that the temperature measured by the sensors which were located at the sides of the hive changed synchronously with the outside temperature. This means that this location is not appropriate for the bee colony behaviour and state identification. This was also observed by other authors, but during the wintering period. Their results showed that the center temperature of overwintering bee flock changed synchronously with the outside temperature (Yu \& Han, 2003).

The monitoring results showed that the temperature measured by the sensor located in the middle of the hive showed a stable temperature between $30^{\circ} \mathrm{C}$ and $35^{\circ} \mathrm{C}$ (Figure 7). This means that this location is appropriate for the bee colony behaviour and state identification. Observed temperature indicates that the bee colony is in an active state and makes brood (this state is also confirmed by the beekeeper visually observing the colony).

Temperature monitoring of the colony placed in the frameless hive also showed similar dynamics comparing to the colony placed in the modern hive (Figure 8). It means, that the basic remote monitoring of the bee colony is also possible for the frameless hives.

\section{Weight monitoring}

Load cell accuracy was verified in the lab during test measurements, comparing the acquired values with the known weight placed on the scales. It should be mentioned that during laboratory experiments with the developed platform, it is found that weight is fluctuating in relation to the environment temperature. When ambient temperature increases, weight of the hive decreases, and by decrease of ambient temperature weight increases. This can be explained by electronics used in the hardware platform and authors made a conclusion that $\mathrm{A} / \mathrm{D}$ converter is affected by the temperature changes. It is concluded that by the change of the temperature to $1^{\circ} \mathrm{C}$ weight is changed up to $20 \mathrm{~g}$.

Figure 9 demonstrates the weight dynamics in Apis Cerana bee colony. It can be observed that during first days (taking into account weight correction) there is no increase in the colony weight. This means that the bee colony is able to forage resources needed only 


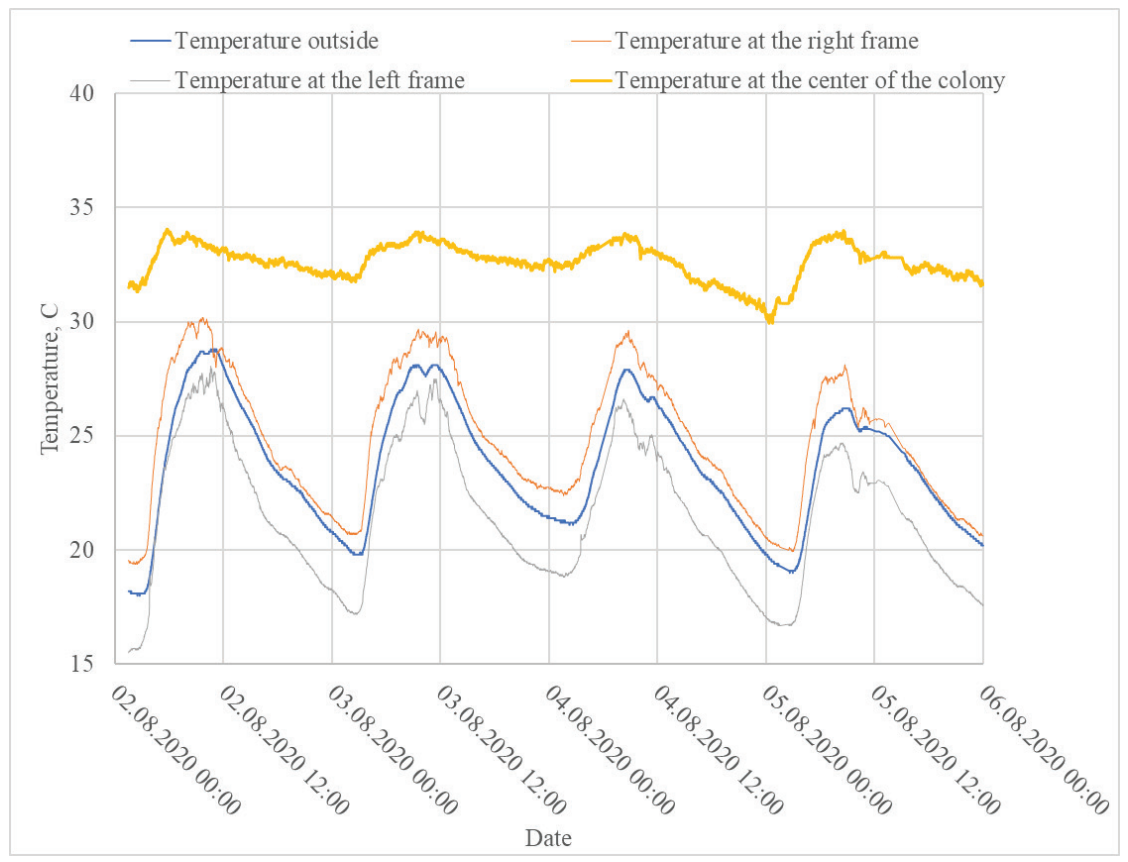

Figure 7. Temperature dynamics in the modern hive.

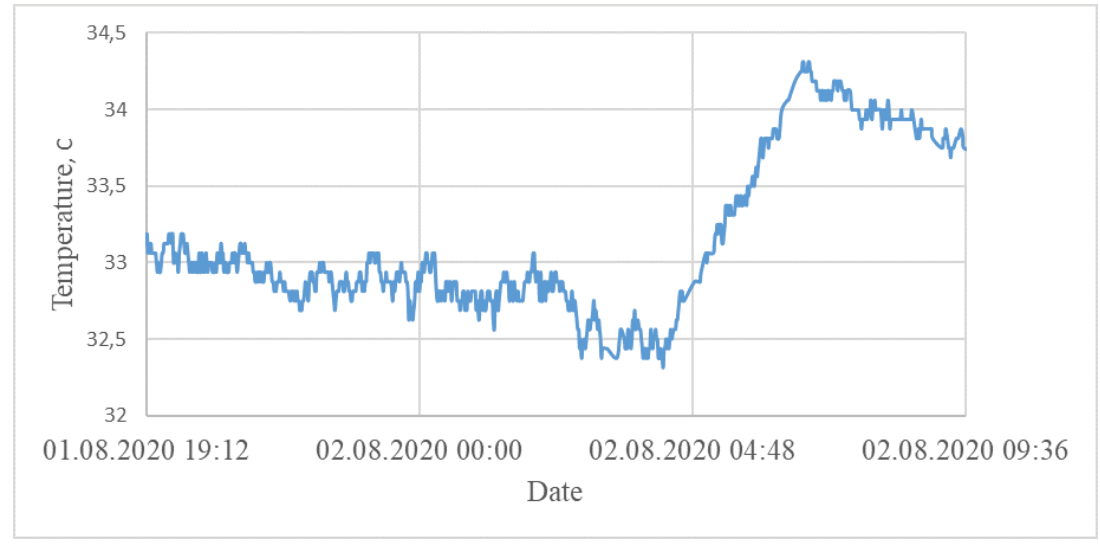

Figure 8. Temperature dynamics in the frameless hive.

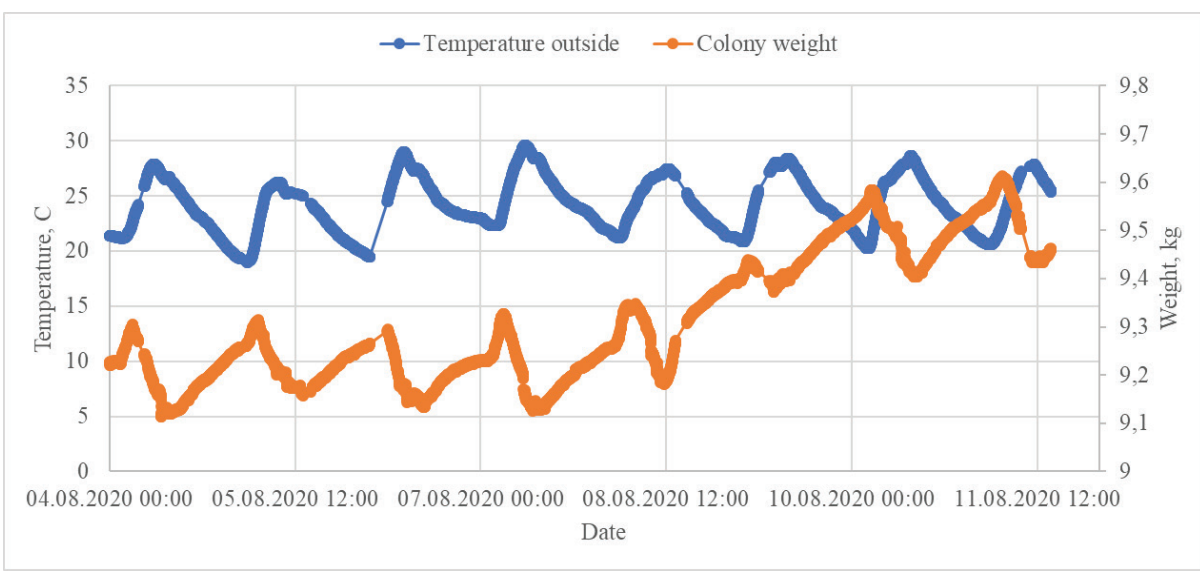

Figure 9. Weight dynamics of the colony. 
for the colony survival. But then a slight increase in weight is observed.

Feedback from the beekeeper

During this study authors also collected a feedback from the private beekeeper about the developed monitoring system and collected data. Some key points addressed by the beekeeper are:

1. The system should be unseen from the outside and hardware parts should be hidden. This is needed to minimise the risk of possible theft.

2. The system installation process is very easy and the beekeeper can deploy the system itself without an assistance from the technical staff.

3. The beekeeper was impressed by the SAMS DW functionality and the possibility to remotely see how the colony is behaving.

4. Monitoring of only the bee colony temperature would not give a big benefit to a full-time beekeeper, rather this feature is mostly valuable if the beekeeper has other full-time jobs (or professions) and other family members help monitor the colony behaviour.

\section{Conclusions}

For the demonstration of the bee colony monitoring procedure in Indonesia, two bee colonies were equipped with the SAMS monitoring devices.

Installation of the precision beekeeping system in Indonesia allows to decrease the number of the visual on-site observations of the bee colonies and helps to detect abnormal behaviour of the colonies and could help to prevent death, absconding, colony collapse disorder (CCD) of the bee colonies.

One temperature sensor can be used for the remote bee colony monitoring. It is concluded that the sensor placement is important for the reliable bee colony temperature collection. The sensor should be placed in the middle of the hive near to the bee cluster, especially if the colony is a small one.

It is approved, that the temperature and weight monitoring can be used also for the frameless colonies, that are placed into simple bee colony trap boxes.

Implementation of the bee colony monitoring system to facilitate the development of the precision beekeeping in Indonesia is necessary and has an added value for the beekeepers, but still transfer of knowledge is needed to facilitate the understandability of the benefits provided by the remote bee colony monitoring systems, including the economic benefits for implementation of such systems.

In relation to the future work, there are several potential directions to work on:

- Monitoring of the Apis Cerana colonies during the whole year to identify the colony development phases;
- Improvement of the monitoring system and potential commercialisation of it;

- Adding additional functionality to the monitoring system, for instance, a solution to detect thefts, as it was mentioned by the beekeepers as important feature;

- Improvements to the developed data warehouse and implementation of analytics models.

\section{References}

Bencsik, M., Bencsik, J., Baxter, M., Lucian, A., Romieu, J., \& Millet, M. (2011). Identification of the honey bee swarming process by analysing the time course of hive vibrations. Computers and Electronics in Agriculture, 76(1), 44-50. https:// doi.org/10.1016/j.compag.2011.01.004

Brodschneider, R., Gray, A., Adjlane, N., Ballis, A., Brusbardis, V., Charrière, J.-D., ... Danihlík, J. (2018). Multi-country loss rates of honey bee colonies during winter 2016/2017 from the COLOSS survey. Journal of Apicultural Research, 57(3), 452-457. https://doi.org/10.108 0/00218839.2018.1460911

Brodschneider, R., Gray, A., van der Zee, R., Adjlane, N., Brusbardis, V., Charrière, J. D., ... Woehl, S. (2016). Preliminary analysis of loss rates of honey bee colonies during winter 2015/16 from the COLOSS survey. Journal of Apicultural Research, 55(5), 375-378. https://doi.org/10.108 0/00218839.2016.1260240

Crane, E. (1990). Bees and beekeeping: science, practice and world resources. Heinemann Newnes.

Delaplane, K. S., Van Der Steen, J., \& Guzman-Novoa, E. (2013). Standard methods for estimating strength parameters of Apis mellifera colonies. Journal of Apicultural Research, Vol. 52. https:// doi.org/10.3896/IBRA.1.52.1.03

Demisew, W. A. (2016). Beekeeping in Ethiopia: Country situation. ApiExpo Africa, 1-32.

Edwards-Murphy, F., Popovici, E., Whelan, P., \& Magno, M. (2015). Development of an heterogeneous wireless sensor network for instrumentation and analysis of beehives. Conference Record - IEEE Instrumentation and Measurement Technology Conference, 2015-July, 346-351. https://doi.org/10.1109/ I2MTC.2015.7151292

Gratzer, K., Susilo, F., Purnomo, D., Fiedler, S., \& Brodschneider, R. (2019). Challenges for Beekeeping in Indonesia with Autochthonous and Introduced Bees. Bee World, 96(2), 40-44. https://doi.org/10.1080/0005772x.2019.1571211

Gray, A., Brodschneider, R., Adjlane, N., Ballis, A., Brusbardis, V., Charrière, J. D., ... Soroker, V. 
(2019). Loss rates of honey bee colonies during winter 2017/18 in 36 countries participating in the COLOSS survey, including effects of forage sources. Journal of Apicultural Research. https:// doi.org/10.1080/00218839.2019.1615661

Guanhuang, Y., Dongjiang, S., Jingcheng, X., Qinghai, S., \& Guilian, L. (1999). Study on the Regulation Ability of Apis cerana cerana on Temperature, Relative Humidity and CO2 Concentration in Its Colony. Scientia Agricultura Sinica, 03.

Hyatt, S. (2012). Asian Honey Bee (Apis cerana javana) in Cairns, Far North Queensland.

Meikle, W. G., \& Holst, N. (2015). Application of continuous monitoring of honeybee colonies. Apidologie, 46(1), 10-22. https://doi. org/10.1007/s13592-014-0298-x

Negash, B., \& Greiling, J. (2017). Quality Focused Apiculture Sector Value Chain Development in Ethiopia. Journal of Agricultural Science and Technology A, 7(2). https://doi. org/10.17265/2161-6256/2017.02.005

Van Der Zee, R., Pisa, L., Andonov, S., Brodschneider, R., Charrière, J. D., Chlebo, R., ... Wilkins, S. (2012). Managed honey bee colony losses in Canada, China, Europe, Israel and Turkey, for the winters of 2008-9 and 2009-10. Journal of
Apicultural Research, 51(1), 100-114. https:// doi.org/10.3896/IBRA.1.51.1.12

Wakjira, K., \& Alemayehu, G. (2019). Assessment of Colony Carrying Capacity and Factors Responsible for Low Production and Productivity of Beekeeping in Horro Guduru Wollega Zone of Oromia, Ethiopia. International Journal of Natural Resource Ecology and Management, 4(1), 14-21. https://doi.org/10.11648/j. ijnrem.20190401.13

Yu, L., \& Han, S. (2003). Overwintering and size dynamic characters of Apis cerana cerana. The Journal of Applied Ecology, 14(5), 721-724.

Zacepins, A., Brusbardis, V., Meitalovs, J., \& Stalidzans, E. (2015). Challenges in the development of Precision Beekeeping. Biosystems Engineering, 130, 60-71. https://doi. org/10.1016/j.biosystemseng.2014.12.001

Zacepins, A., Kviesis, A., Ahrendt, P., Richter, U., Tekin, S., \& Durgun, M. (2016). Beekeeping in the future - smart apiary management. ICCC 2016, 808-812.

Zacepins, A., Kviesis, A., Komasilovs, V., \& Muhammad, F. R. (2020). Monitoring System for Remote Bee Colony State Detection. Baltic J. Modern Computing, 8(3), 461-470.

\section{Acknowledgments}

Scientific research, publication and presentation are supported by the Horizon 2020 Project SAMS 'Smart Apiculture Management Services'. This project receives funding from the Horizon 2020 European Union Research and Innovation Framework under Grant Agreement Nr.780755 - SAMS.

We would like to extend our gratitude to Aditya Rizky Pratama from Labtek Indie for providing high quality pictures of the beehives and monitoring system installation process. 